\title{
Improving Energy Usage in Energy Harvesting Wireless Sensor Nodes Using Weather Forecast
}

\author{
Strahinja Janković, Student Member, IEEE, Dragomir El Mezeni, \\ and Lazar Saranovac, Member, IEEE
}

\begin{abstract}
Battery powered wireless sensor nodes are used in many applications. They can be placed in remote locations and can operate for a long period of time without the need for maintenance. However, finite battery lifetime is one of the main limitations of such devices. Energy harvesting can be used to recharge batteries from environmental energy sources. This enables continuous work when energy neutrality is satisfied. For optimal use of available energy, when energy harvesting is used, the optimization goal switches from energy management to workload maximization while maintaining energy neutrality. In order to achieve energy neutrality, prediction of energy that can be harvested in the future is needed. This prediction can be based on previous measured data. However, this approach can be unreliable when weather conditions change during the day or between days. To improve prediction precision, weather forecast can be used. This information has been used to predict energy that can be harvested in the future but only for the next few hours. We present a twolevel predictor that uses cloud cover information from hourly weather forecast for next $\mathbf{2 4}$ hour period to predict energy that can be harvested in the same time interval. Proposed predictor achieves a $26 \%$ less prediction mean absolute percentage error, a $15 \%$ less mean absolute deviation percent error and allows an $8 \%$ better performance of simulated wireless sensor node compared to Exponentially Weighted Moving Average (EWMA) predictor.
\end{abstract}

Keywords - Energy Harvesting, Energy Neutrality, Power Management, Weather Forecast, Wireless Sensor Node.

\section{INTRODUCTION}

$\mathrm{B}$ ATTERY powered embedded systems with wireless connection capability are widespread in everyday life. In recent years, Internet of Things (IoT) [1] has gained a lot of popularity as an increasing number of connected devices are introduced. It is expected that by the year 2020

Paper received April 26, 2018; revised June 22, 2018; accepted June 23, 2018. Date of publication July 31, 2018. The associate editor coordinating the review of this manuscript and approving it for publication was Prof. Vujo Drndarević.

This paper is a revised and expanded version of the paper presented at the 25th Telecommunications Forum TELFOR 2017 [25].

This work was supported by the Serbian Ministry of Education, Science and Technological Development through the project TR32043.

Corresponding author Strahinja Janković is Ph.D. candidate at the School of Electrical Engineering, University of Belgrade, Bul. kralja Aleksandra 73, 11120 Belgrade, Serbia (e-mail: jankovics@etf.bg.ac.rs).

Strahinja Janković, Dragomir El Mezeni and Lazar Saranovac are with the School of Electrical Engineering, University of Belgrade, Bul. kralja Aleksandra 73, 11120 Belgrade, Serbia. the number of connected devices will be between 20 and 30 billion [2].

A number of battery powered embedded systems with sensing and actuating capability can be organized into a Wireless Sensor Network (WSN). A WSN can consist of many densely grouped wireless sensor nodes, or nodes that are placed in remote and inaccessible locations [3]. Finite battery lifetime of wireless sensor nodes is a limitation which affects the operation of WSN. If a wireless sensor node's battery gets depleted WSN operation can be compromised.

Power/energy management is used to prolong battery life of battery powered embedded systems at the cost of performance. For example, energy efficient protocols can significantly reduce communication power consumption, which is the most critical part, especially in WSNs [4]. Also, all sorts of power management techniques, such as voltage and frequency scaling can be used to reduce data processing power consumption. Energy-aware data acquisition can reduce power consumption of sensing subsystem [5]. However, regardless of how low power consumption is the battery will eventually get depleted. Replacing battery may not be possible if WSN nodes are inaccessible, or isn't cost effective if the number of nodes is too large.

One solution is to use rechargeable batteries and energy harvesting to recharge them. There are many environmental energy sources suitable for harvesting, for example solar, wind, vibration, RF. These energy sources can be classified based on their controllability and predictability. For instance, solar energy is uncontrolled, but can be predicted because of its diurnal cycle [6].

With energy harvesting, battery powered system optimization goal shifts from reducing energy consumption to maximizing workload, while maintaining operation without interruption. This concept is also known as energy neutrality [7].

Energy neutrality can be achieved if information about both currently stored energy and energy that can be harvested in the future is available. In order to estimate energy that can be harvested in the future, different prediction techniques have been used. They can be classified into two categories: past predicts the future (PPF) and weather forecast-based techniques [8].

In PPF techniques, information about previous available samples is used to predict energy that can be harvested in the future. All of these techniques split the day into several, equally sized, time slots. Prediction is usually 
done for the next time slot in the same day or for the same time slot in the following day. PPF techniques are not computationally complex, but they produce a significant prediction error when weather changes during the day, or between consecutive days. Also, they cannot give a longterm prediction for more than 24 hours ahead.

Exponentially Weighted Moving Average (EWMA) [7] estimates the amount of energy that can be harvested during a certain time of the day as a weighted average of harvested energy from previous days. It doesn't require any additional memory for historical data and can facilitate adaptation to seasonal changes. However, since it is essentially a low-pass filter, EWMA exhibits large prediction errors when there are sudden changes in weather conditions. To cope with this, Weather Conditioned Moving Average (WCMA) [9], is adapting EWMA-based weights according to the weather conditions in the current day. Changes in weather conditions are detected as large values of ratio between currently harvested energy and mean value obtained from the previous samples. Large values of this ratio imply lower importance of historical data, since weather conditions have changed. Hence, weights in estimator are adjusted to enable faster adaptation to new conditions. Although it enables faster adaptation to the changing weather conditions, WCMA provides predictions only for the next few time slots. ProEnergy [10] also offers shortterm predictions similar to EWMA and WCMA, but unlike them it also provides medium-term energy predictions by exploring correlation between different samples. Accurate Solar Energy Allocation (ASEA) [11] represents an algorithm for energy consumption optimization, which aims at maximal utilization of environmental energy, while avoiding battery depletion. For estimation of energy that will be gathered in the next time slot it uses an algorithm similar to WCMA. There have also been attempts at using regression analysis [12], Q-Learning [13], and piecewise least squares curve fitting [14].

To increase prediction precision weather forecast can be used, often together with past data, to predict energy that can be harvested in the future. It has been shown [8] that weather forecast-based prediction can give less prediction error than PPF in periods from 3 hours to few days ahead. Authors in [15] have used weather forecast combined with historical data from Korea Metrological Administration to predict energy that can be harvested in the following 3 hours. Authors in [16] have used weather forecast-based technique presented in [8] and applied it to task scheduling. Weather forecast information has also been used for task-scheduling in [17] for a FPGA-based wireless sensor node which supports hardware reconfiguration at runtime. However, all research so far has been focused on short-term future prediction intervals.

The goal of this work was to use weather forecast to predict energy that can be harvested in the next 24 hours in order to optimize energy usage and achieve energy neutrality. If energy that can be harvested during the following day is enough to recharge energy storage, then more energy can be used during the night even if current weather conditions are not favorable. In the same manner, if current conditions are good, but forecast for the following day is not favorable, energy should be stored so performance in the following day is not compromised. Weather forecast data request and processing is done on a server in the cloud or on the gateway node and target node only receives processed data.

The paper is organized as follows. Details about weather forecast information and solar radiation are presented in Section 2. In Section 3 the proposed model is presented. Results and discussion are in Section 4 and conclusion is in Section 5.

\section{WEATHER FORECAST}

Weather forecasts are available online from many providers, with different parameters being tracked and forecasted. A parameter that is related to available solar energy is cloudiness or cloud cover [18]. It is usually given in oktas, which represent how many eights of the sky are covered with clouds, going from 0 (no clouds) to 8 (complete sky covered with clouds), or in percentage of sky that is covered with clouds.

Many global weather forecast providers give a prediction of future cloud cover. In this paper Dark Sky [19] was used. It provides hourly weather forecast, including cloud cover for up to 7 days ahead and historical weather data. Data is requested using HTTP GET method formatted according to supported API requests [20]. Response is in JavaScript Object Notation (JSON) format.

In order to obtain information about energy that can be harvested in the future from cloud cover data, information about solar irradiance for a desired geographical location is needed. Solar irradiance data can be obtained from PVGIS [21]. PVGIS provides historical hourly solar irradiance data and average day in a month solar irradiance data. Global solar irradiance data is separated into diffuse and direct components. Direct irradiance depends on cloud cover and has the largest intensity when there are no clouds. Diffuse irradiance is present even if sky is completely covered with clouds.

Based on forecasted cloud cover and average daily solar irradiance data from PVGIS, forecasted solar irradiance $S I_{F C}$ is

$$
S I_{F C}=S I_{D I F F}+\left(1-C C_{F C}\right) S I_{D I R}
$$

where $S I_{D I F F}$ is average diffuse irradiance, $C C_{F C}$ is forecasted cloud cover and $S I_{D I R}$ is average direct irradiance.

Average global solar irradiance for Belgrade in January, May and September obtained from PVGIS is shown in Fig. 1.

\section{PROPOSED MODEL}

System model is shown in Fig. 2. System consists of energy generator (solar panel), energy storage (battery or super capacitor), load which consumes energy and manager which implements prediction technique and 


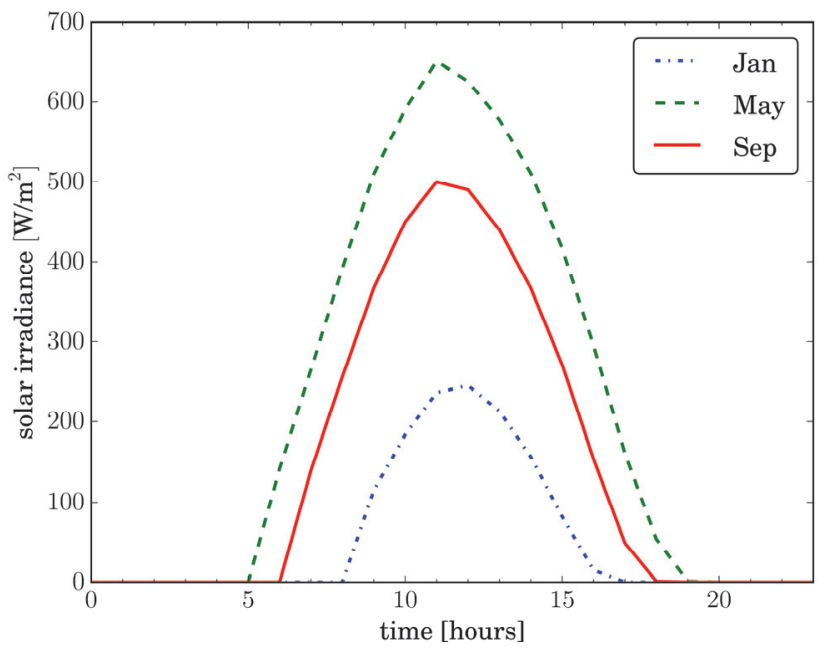

Fig. 1. PVGIS average daily solar irradiance for Belgrade in January, May and September.

configures load in order to maximize performance. Between energy generator and energy storage, and energy storage and load there are non-ideal converters with $\eta_{1}$ and $\eta_{2}$ efficiencies.

\section{A. Energy generator}

Energy is generated using a solar panel. Solar panel model is based on panel size $110 \times 60 \mathrm{~mm}$ and $15 \%$ efficiency. Energy that is harvested $H E$ is

$$
H E=\text { Area } \cdot \text { Eff } \cdot S I
$$

where Area is solar panel area, Eff is solar panel efficiency and $S I$ is current solar irradiance.

\section{B. Energy storage}

Energy storage is modeled as an $100 \mathrm{~F} 2.7 \mathrm{~V}$ ideal super capacitor which can store energy equivalent to an 100 mAh 3.7V LiPo battery. Efficiency of energy conversion from energy generator to energy storage $\eta_{1}$ is set to $85 \%$.

\section{Load}

Load is a CC430 wireless sensor node modeled in our previous paper [22]. It has $60 \mathrm{~mW}$ average power consumption when transmitting data with $0 \mathrm{dBm}$ transmission power and $0.015 \mathrm{~mW}$ average power consumption when in LPM3 low power mode. Based on currently available and predicted energy that can be harvested in the future, load is duty cycled. The sample rate and number of transmitted bytes are increased when there is more energy available and reduced when energy resources are scarce. Efficiency of energy conversion from energy storage to load $\eta_{2}$ is set to $95 \%$.

\section{Manager}

Manager uses weather forecast data to predict the amount of energy that will be harvested in the future time intervals. Weather forecast data is obtained every day at noon, since it is expected that the most solar energy will be available at that time. Hourly forecast is taken for 24

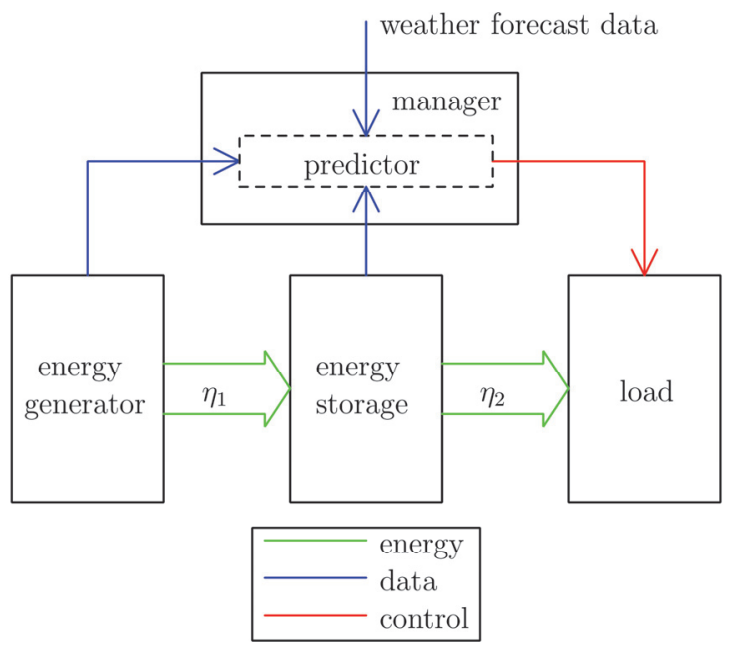

Fig. 2. Proposed system model.

hours in advance. This way the overhead of obtaining weather forecast is minimized.

Prediction is implemented in two levels. Forecast data is used to generate the initial prediction of solar energy that can be harvested in the following 24 hours. Since hourly weather forecast is used, a day is divided into 24 time slots. Prediction is refined by using a relative difference between generated and predicted energy for a previous time slot to update prediction for the following time. If $E_{F C}^{(i)}$ is weather forecast-based prediction of energy that will be harvested in time slot $i$ and $E_{\text {harv }}^{(i)}$ is actual harvested energy in time slot $i$ then the prediction of generated energy in time slot $i, \widetilde{E}^{(i)}$, is

$$
\begin{gathered}
\widetilde{E}^{(i)}=(1+\alpha) \cdot E_{F C}^{(i)}, \\
\alpha=\frac{E_{\text {har }}^{(i-1)}-E_{F C}^{(i-1)}}{E_{F C}^{(i-1)}}, \alpha \in[-0.3,0.05] .
\end{gathered}
$$

Update is limited to an increase of $5 \%$ or reduction of $30 \%$ since we are trying to minimize overestimation in prediction.

Based on the prediction of solar energy that can be harvested in the future, load is configured in order to maximize performance. A maximum duty cycle of load is set to $D C_{M A X}$ and energy consumed with $D C_{M A X}$ duty cycle during single time slot is $E_{L M A X}$. As long as predicted generated energy in the next time slot is higher than $E_{L M A X}$, load duty cycle is set to $D C_{M A X}$. This is illustrated in Fig. 3 where duty cycle is set to $D C_{M A X}$ for time slots $[0 . . i-1]$ and $[j+1 . . N-1]$. In our experiments, $D C_{M A X}$ is set to $40 \%$.

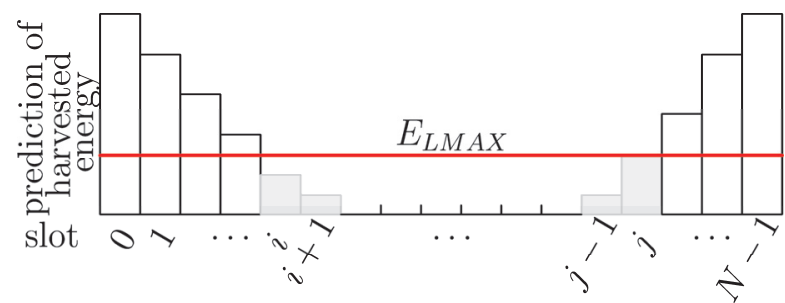

Fig. 3. Prediction of harvested energy in time slots. 
Once predicted generated energy falls below $E_{L M A X}$, forecast data for future time slots is traversed until a time slot with predicted generated energy higher than $E_{L M A X}$ is found. In Fig. 3 those are time slots $[i . . j]$. Total predicted generated energy in time period $[i . . j]$ together with battery state of charge at time slot $i, B_{S o C}^{(i)}$, is divided by the size of this time period to obtain target average power consumption in each time slot $P_{A V G}$

$$
P_{A V G}=\frac{B_{S o C}^{(i)}+\sum_{k=i}^{j} \widetilde{E}^{(k)}}{T \cdot(j-i)},
$$

where $T$ is length of a time slot.

Using $P_{A V G}$ duty cycle $D C_{n i g h t}$ is

$$
D C_{n i g h t}=\frac{K \cdot P_{A V G}-P_{O F F}}{P_{O N}-P_{O F F}},
$$

where $K$ is a correction factor, $P_{O F F}$ is average load power consumption when it is inactive and $P_{O N}$ is average load power consumption when it is active. Correction factor $K$ is introduced to prevent prediction overestimates. Namely, in an ideal case, $K$ can be set to 1 , which would mean that battery gets fully depleted over the night at the exact time when sun starts rising. As the battery starts charging with the sunrise, load actually never stops working. However, since predictions are not ideal, it can happen that a battery gets depleted before sunrise, leaving no energy for load functioning. To prevent this and to maintain energy neutrality, a correction factor must be set to the value lower than 1 . In our experiments $K$ is set to 0.5 making the proposed algorithm more robust to prediction errors.

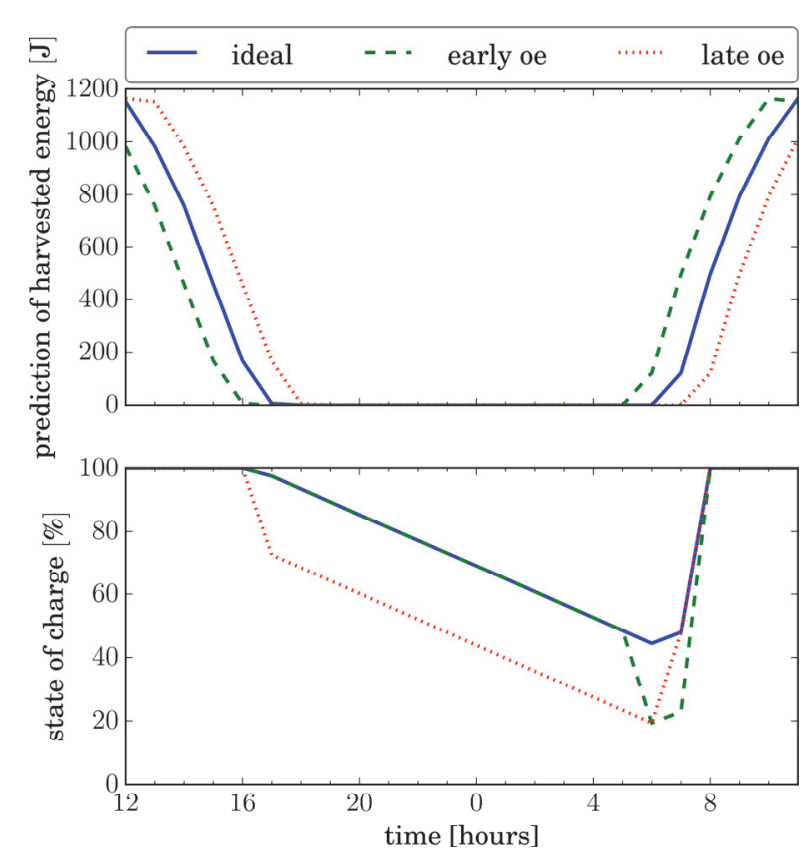

Fig. 4 Effects of prediction accuracy on battery state of charge: ideal prediction, overestimation early in the day and overestimation late in the day.

\section{RESUlTS AND DISCUSSION}

In Fig. 4 three different predictions and a resulting battery state of charge are shown for a 24 hour period starting from noon. It can be noticed that in all three cases, battery recharges in an hour or two since sunrise and it stays at the maximum value until the sunset. The ideal prediction is the prediction that matches actual generated energy. In this case, battery state of charge goes down to around $50 \%$ when sun rises and starts charging battery. If prediction overestimates available energy at the start of the day, then an increased duty cycle will rapidly start to reduce battery state of charge before sunrise. If prediction overestimates available energy at the end of the day, then an increased duty cycle will reduce state of charge before sunset and battery may get depleted before sunrise the following day. From this analysis we can notice that the accuracy of predictions is critical at both ends of the solar cycle, at sunrise and sunset.

Proposed model has been implemented and tested in a simulated environment, while using publicly available real-world weather data. Weather data including solar radiation from Košutnjak automatic weather station [23] and hourly cloud cover forecast from Dark Sky [19] have been gathered for two months in the period from August 21 st to October 20th 2017.

The performance of proposed model is compared to EWMA [7]. Actual solar energy, calculated from measured solar radiation for two weeks, is compared to predictions obtained using EWMA and proposed twolevel predictor. These results are presented in figures 5a and 5b. Prediction errors are calculated in two ways: Mean Absolute Percentage Error (MAPE) and Mean Absolute Deviation Percent (MADP). MAPE is calculated as

$$
M A P E=\frac{100}{N} \sum_{i}\left|\frac{E_{\text {harv }}^{(i)}-\widetilde{E}^{(i)}}{E_{\text {harv }}^{(i)}}\right|,
$$

while MADP is calculated as

$$
M A D P=100 \cdot\left|\frac{E_{H}-\widetilde{E}_{H}}{E_{H}}\right|,
$$

where $E_{H}$ is harvested energy in an arbitrary number of consecutive time slots and $\widetilde{E}_{H}$ is predicted energy in the same time slots. MAPE is commonly used for comparing predictor quality. However, this metric exhibits large influence of the time slots with low harvested energy. Because of that, these time slots need to be filtered out [24]. MADP is regarded as more robust metric since it doesn't have the previously mentioned problem. To facilitate easier comparison with available prediction techniques, both results are given. Both errors are calculated for the whole 61 day period.

TABLE 1: COMPARISON OF PROPOSED TWO-LEVEL PREDICTOR AND EWMA.

\begin{tabular}{l|rrr}
\hline Predictor & $\boldsymbol{M A P E}$ & $\boldsymbol{M A D P}$ & $\boldsymbol{D C}_{\boldsymbol{A} \boldsymbol{V} \boldsymbol{G}}[\%]$ \\
\hline Proposed two-level & 20.0 & 18.0 & 21.4 \\
EWMA & 27.3 & 21.2 & 19.8 \\
\hline
\end{tabular}



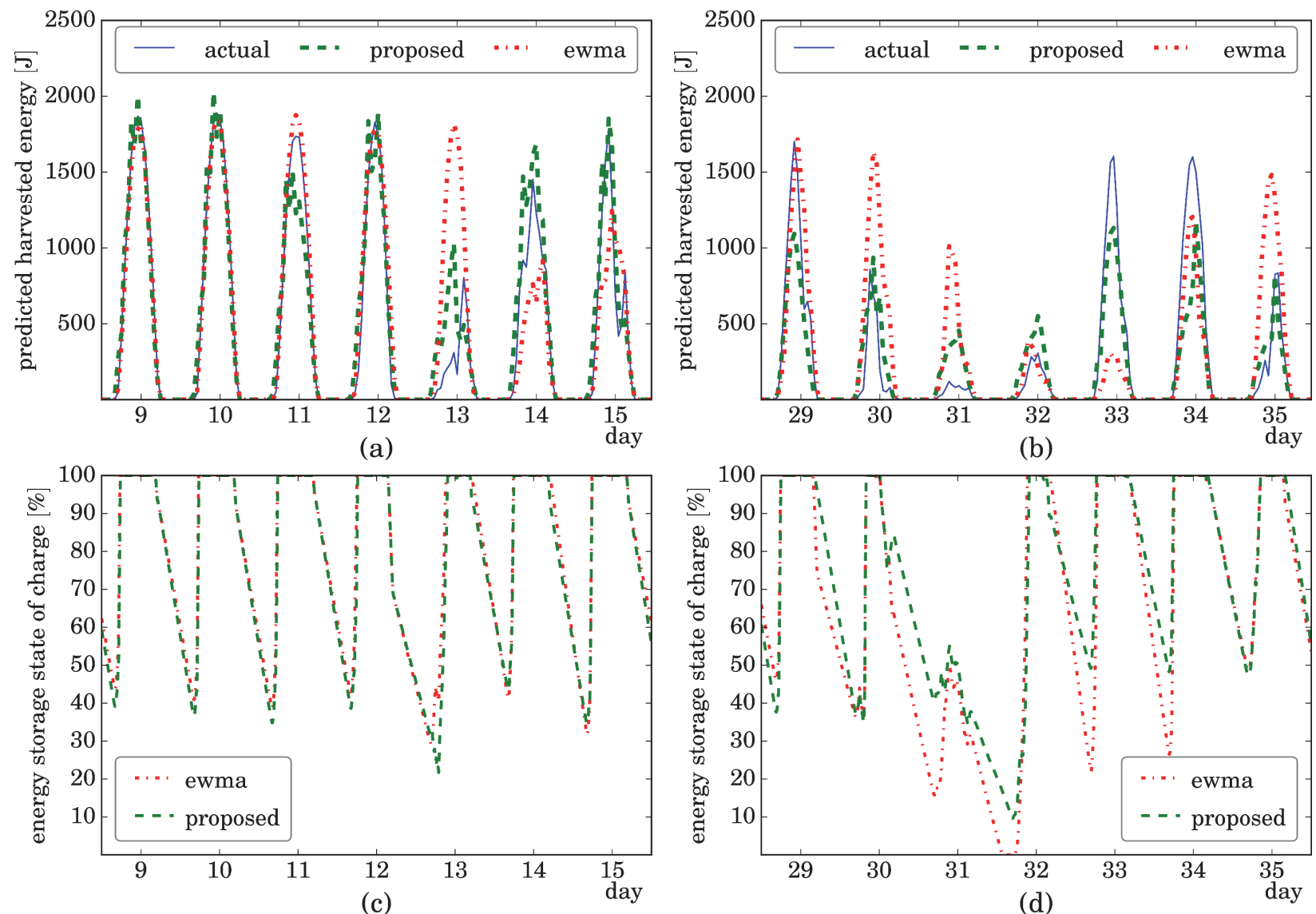

Fig. 5. Comparison of proposed model and EWMA: a) Predicted harvested energy on days 9 to 15; b) Predicted harvested energy on days 29 to 35; c) Energy storage state of charge on days 9 to 15; d) Energy storage state of charge on days 29 to 35 .

Looking at days 12 to 14 in Fig. 5a and 29 to 33 in Fig. $5 \mathrm{~b}$ it can be seen that EWMA prediction error increases when cloudy and sunny days go one after another. This is especially problematic when a cloudy day comes after several sunny days because energy that can be harvested in the future is overestimated, which can lead to battery depletion.

Energy storage state of charge for two weeks of data is shown in figures $5 \mathrm{c}$ and $5 \mathrm{~d}$. When there is enough harvested energy during the day, storage is fully charged. During the night energy storage is discharged until sun rises again. Looking at figures $5 \mathrm{c}$ and $5 \mathrm{~d}$ it is visible that energy storage gets depleted several times when using EWMA predictor, because of overestimate when a cloudy day follows several sunny days. On the other hand, energy storage is never depleted when the proposed predictor is used.

The amount of work that is done using proposed twolevel predictor and EWMA is shown in table 1. Proposed model allows $8 \%$ more work to be done while maintaining energy neutrality.

The performance of proposed two-level predictor has also been compared to WCMA [9]. WCMA performs better in terms of prediction error, with $\mathrm{MAPE}=3.0 \%$ and $\mathrm{MADP}=2.3 \%$. However, since WCMA can be used only for prediction of energy in next time slot and not for 24 hours ahead, it could not be applied to the model used in this work.

\section{CONCLUSION}

In this paper a two-level weather forecast based solar radiation prediction model is presented. Model achieves 8\% better performance than EWMA with 24-hour predictions. Performance of the model depends on the quality of weather forecast and can be further improved.

Presented two-level predictor has negligible performance overhead since weather information data is obtained only once per day from a gateway node, when there is maximum solar energy available.

Weather forecast prediction can be further improved by better fusion of weather forecast and previous data, combining several forecasts to reduce error rate or by extending forecast period to more than 24 hours.

Manager optimization algorithm that was used in this paper was optimized for simulated setup and is highly dependent on the characteristics of used components: solar panel area and efficiency, energy storage capacity and load power consumption. Further research should be directed to exploring how different setup configurations affect the performance of proposed predictor and attempt to generalize solution. 


\section{REFERENCES}

[1] L. Atzori, A. Iera, and G. Morabito, "The Internet of Things: A survey," Computer Networks, vol. 54, no. 15, pp. 2787-2805, Oct. 2010 .

[2] A. Nordrum, "Popular Internet of Things Forecast of 50 Billion Devices by 2020 Is Outdated," IEEE Spectrum: Technology, Engineering, and Science News, 18-Aug-2016. [Online]. Available: http://spectrum.ieee.org/tech-talk/telecom/internet/popular-internetof-things-forecast-of-50-billion-devices-by-2020-is-outdated.

[3] I. F. Akyildiz, W. Su, Y. Sankarasubramaniam, and E. Cayirci, "Wireless sensor networks: a survey," Computer Networks, vol. 38, no. 4, pp. 393-422, Mar. 2002.

[4] G. Anastasi, M. Conti, M. Di Francesco, and A. Passarella, "Energy conservation in wireless sensor networks: A survey," Ad Hoc Networks, vol. 7, no. 3, pp. 537-568, May 2009.

[5] C. Alippi, G. Anastasi, M. Di Francesco, and M. Roveri, "Energy management in wireless sensor networks with energy-hungry sensors," IEEE Instrumentation \& Measurement Magazine, vol. 12, no. 2, pp. 16-23, Apr. 2009.

[6] S. Sudevalayam and P. Kulkarni, "Energy Harvesting Sensor Nodes: Survey and Implications," IEEE Communications Surveys Tutorials, vol. 13, no. 3, pp. 443-461, Third 2011.

[7] A. Kansal, J. Hsu, S. Zahedi, and M. B. Srivastava, "Power Management in Energy Harvesting Sensor Networks," ACM Trans. Embed. Comput. Syst., vol. 6, no. 4, Sep. 2007.

[8] N. Sharma, J. Gummeson, D. Irwin, and P. Shenoy, "Cloudy Computing: Leveraging Weather Forecasts in Energy Harvesting Sensor Systems," in 2010 7th Annual IEEE Communications Society Conference on Sensor, Mesh and Ad Hoc Communications and Networks (SECON), 2010, pp. 1-9.

[9] J. R. Piorno, C. Bergonzini, D. Atienza, and T. S. Rosing, "Prediction and management in energy harvested wireless sensor nodes," in 2009 1st International Conference on Wireless Communication, Vehicular Technology, Information Theory and Aerospace Electronic Systems Technology, 2009, pp. 6-10.

[10] A. Cammarano, C. Petrioli, and D. Spenza, "Pro-Energy: A novel energy prediction model for solar and wind energy-harvesting wireless sensor networks," in 2012 IEEE 9th International Conference on Mobile Ad-Hoc and Sensor Systems (MASS 2012), 2012, pp. 75-83.

[11] D. K. Noh and K. Kang, "Balanced energy allocation scheme for a solar-powered sensor system and its effects on network-wide performance," Journal of Computer and System Sciences, vol. 77, no. 5, pp. 917-932, Sep. 2011.

[12] J. Lu, S. Liu, Q. Wu, and Q. Qiu, "Accurate modeling and prediction of energy availability in energy harvesting real-time embedded systems," in International Conference on Green Computing, 2010, pp. 469-476.

[13] S. Kosunalp, "A New Energy Prediction Algorithm for EnergyHarvesting Wireless Sensor Networks With Q-Learning," IEEE Access, vol. 4, pp. 5755-5763, 2016.

[14] T. Zou, S. Lin, Q. Feng, and Y. Chen, "Energy-Efficient Control with Harvesting Predictions for Solar-Powered Wireless Sensor Networks," Sensors, vol. 16, no. 1, p. 53, Jan. 2016.

[15] K. Kwon, J. Yang, and Y. Yoo, "Adaptive Control of the Packet Transmission Period with Solar Energy Harvesting Prediction in Wireless Sensor Networks," Sensors, vol. 15, no. 5, pp. 9741-9755, Apr. 2015.

[16] Y. Li, Z. Jia, and X. Li, "Task Scheduling Based on Weather Forecast in Energy Harvesting Sensor Systems," IEEE Sensors Journal, vol. 14, no. 11, pp. 3763-3765, Nov. 2014.

[17] Y. Li, J. Si, and S. Ma, "Using Energy-aware Scheduling Weather Forecast based Harvesting for Reconfigurable Hardware," IEEE Transactions on Sustainable Computing, pp. 1-1, 2018.

[18] Huschke, Ralph E. (1959) Glossary of Meteorology, American Meteorological Society, Boston, Second printing-1970.

[19] Dark Sky, "Powered by Dark Sky" [Online]. Available: https://darksky.net/poweredby/

[20] Dark Sky, "Dark Sky API - Overview" [Online]. Available: https://darksky.net/dev/docs

[21] Huld T., Müller R. and Gambardella A., 2012: "A new solar radiation database for estimating PV performance in Europe and Africa". Solar Energy, 86, 1803-1815.

[22] S. Janković and L. Saranovac, "High-level Power Modeling of CC430 SoC", in Proceedings of IcETRAN-2017, 2017, Kladovo, Serbia, ELI3.2

[23] Republic Hydrometeorogical Service of Serbia, Meteorological station Beograd - Automatic weather station Košutnjak [Online] http://hidmet.gov.rs/eng/osmotreni/kosutnjak.php

[24] A. Cammarano, C. Petrioli, and D. Spenza, "Online Energy Harvesting Prediction in Environmentally Powered Wireless Sensor Networks," IEEE Sensors Journal, vol. 16, no. 17, pp. 6793-6804, Sep. 2016.

[25] S. Janković and L. Saranovac, "Improving energy usage in energy harvesting wireless sensor nodes using weather forecast," 201725 th Telecommunication Forum (TELFOR), Belgrade, 2017, pp. 1-4. 